\title{
Hepatocyte growth factor leads to recovery from alcohol-induced fatty liver in rats
}

\author{
Minoru Tahara, ${ }^{1}$ Kunio Matsumoto, ${ }^{1}$ Toshihiro Nukiwa, ${ }^{2}$ and Toshikazu Nakamura ${ }^{1}$ \\ ${ }^{1}$ Division of Biochemistry, Department of Oncology, Biomedical Research Center, Osaka University Medical School, Suita, \\ Osaka 565-0871, Japan \\ ${ }^{2}$ Department of Respiratory Oncology and Molecular Medicine, Institute of Development, Aging, and Cancer, Tohoku University, \\ Sendai, Miyagi 980-0872, Japan
}

Address correspondence to: Toshikazu Nakamura, Division of Biochemistry, Department of Oncology, Biomedical Research Center, Osaka University Medical School, Yamadaoka 2-2, Suita, Osaka 565-0871, Japan. Phone: 81-6-679-3783; Fax: 81-6-679-3789; E-mail: nakamura@onbich.med.osaka-u.ac.jp

Received for publication June 30, 1998, and accepted in revised form December 15, 1998.

\begin{abstract}
A fatty liver is characterized by the hyperaccumulation of lipids within hepatocytes and is often caused by excessive alcohol intake. Rats fed ethanol-containing diets for 37 days showed remarkable increase in hepatic lipids and lipid droplet accumulation in the hepatocytes, indicating the onset of alcoholic fatty liver. Administration of hepatocyte growth factor (HGF) for the last seven days of ethanol treatment markedly decreased hepatic lipids to a level lower than that seen before HGF treatment. In contrast, serum levels of lipids and lipoproteins increased with HGF administration. Primary cultured hepatocytes prepared from the fatty liver retained lipid droplets during a 48-hour culture. However, when cultured in the presence of HGF, intracellular lipid concentrations decreased and lipid secretion was enhanced. Consistent with these events, HGF stimulated the rate of protein synthesis of apolipoprotein $\mathrm{B}(\mathrm{apoB})$ and enhanced subsequent mobilization of lipids into the medium. These results indicate that HGF administration induced recovery from the fatty liver, at least in part, by enhancing apoB synthesis and the subsequent mobilization of lipids from hepatocytes with fatty change. The possibility that HGF can be therapeutic for subjects with an alcohol-related fatty liver warrants further attention.
\end{abstract}

J. Clin. Invest. 103:313-320 (1999).

\section{Introduction}

Fatty liver, characterized by hyperaccumulation of lipids in the liver, is often caused by excessive alcohol intake or by hepatotoxic drugs. It has been suggested repeatedly that patients with fatty liver are more susceptible to fibrotic liver diseases (1). The severity of steatosis seen on the initial biopsy predicted the development of liver cirrhosis evidenced in a subsequent biopsy, regardless of the level of continuous alcohol intake (2). Patients with an alcoholic fatty liver who continue drinking alcohol are at a high risk for disease progression (3), and increased collagen synthesis and turnover occur in an alcoholic fatty liver $(4,5)$. Therefore, recovery from a fatty liver seems to simply decrease the susceptibility to liver fibrosis or cirrhosis that are more severe liver diseases in terms of liver functions and treatments.

Hepatocyte growth factor (HGF), originally identified and cloned as a potent mitogen for hepatocytes $(6-8)$, exhibits pleiotropic activities in a wide variety of cells and tissues (9-12). In the liver, HGF plays pivotal roles for development and regeneration of the liver. Essential roles of HGF in liver development were defined by targeted disruption of the HGF gene (13). Physiologically, HGF plays a hepatotrophic role for regeneration of the liver and HGF stimulates regeneration of the liver after various types of liver injuries $(11,12,14-18)$. HGF has anti-apoptotic and/or cytoprotective effects on hepatocytes $(14,17,19)$ and exhibits anti-fibrotic action for liver, fibrosis/cirrhosis, in experimental animals (20-22).
In our studies done to determine the efficacy of HGF on liver fibrosis/cirrhosis, we found that administration of HGF prevented the onset of hepatic steatosis in rat liver (22). Our findings suggested that HGF might affect lipid metabolism and/or lipid mobilization in the injured livers of rats. In this study, we obtained evidence that HGF affected lipoprotein synthesis and mobilization of lipids in hepatocytes, and the alcoholrelated fatty liver showed much improvement when HGF was administered.

\section{Methods}

Animal treatment. Human recombinant HGF (hrHGF) was prepared as described elsewhere $(14,20)$. Four-week-old male Sprague-Dawley rats housed in temperature- and light-controlled rooms were randomly assigned to four groups: (a) rats fed ethanol-containing liquid diets for 30 days $(n=4)$ (23) (Oriental Yeast. Co., Tokyo, Japan); (b) rats pair-fed isocaloric liquid diets without ethanol for 30 days $(n=4)$; (c) rats fed ethanolcontaining liquid diets for 37 days and then intravenously injected daily with saline during the last 7 days $(n=6) ;(d)$ rats fed ethanol-containing liquid diets for 37 days and given daily intravenous injections of $200 \mu \mathrm{g}(n=6)$ or $800 \mu \mathrm{g}(n=6)$ of hrHGF per kilogram of body weight during the last 7 days.

The calorie distribution of liquid diets component is as follows: $16 \%$ as protein, $36 \%$ as fat, $13 \%$ as carbohydrate, and $35 \%$ as either ethanol or additional carbohydrate in the isocaloric liquid diets. The fatty acid composition of both ethanol-containing and isocaloric liquid diets consisted of $33.45 \%$ saturated fat, $66.26 \%$ unsaturated fat, and $0.29 \%$ unknown fat. Ethanol was incorporated into the liquid diets containing all required nutrients, and liquid diets were the only source of fluid and food provided, as 


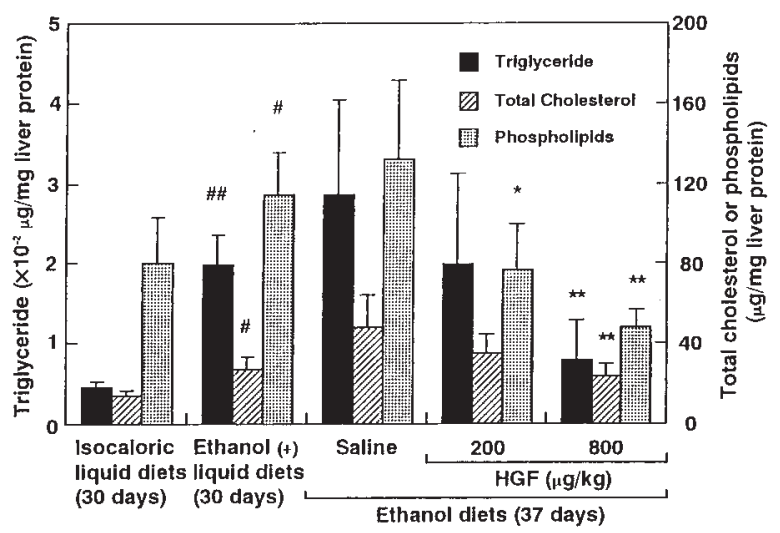

Figure 1

HGF-induced changes in hepatic lipid contents in rats with alcoholic fatty liver. Rats were pair-fed isocaloric liquid diets for 30 days $(n=4)$, or ethanol-containing diets for 30 days $(n=4)$ or 37 days. $\operatorname{hrHGF}(n=6)$ or saline $(n=6)$ alone was daily injected for the last 7 days during a 37-day treatment with ethanol diets. Each value represents the mean \pm SD. $\# P<$ $0.05,{ }^{\# \#} P<0.01$ ethanol-fed rats vs. pair-fed rats; ${ }^{*} P<0.05,{ }^{* *} P<0.01$ HGF-treated rats vs. saline control. HGF, hepatocyte growth factor; hrHGF, human recombinant hepatocyte growth factor.

recommended by the manufacturer. Rats given isocaloric liquid diets without ethanol were pair-fed daily on an isoenergetic basis with the corresponding littermates fed the ethanol-containing diets. Before sacrifice, the rats were fasted overnight but had free access to drinking water for 14-16 h. For analysis of hepatic HGF contents, the rats were fed ethanol-containing or isocaloric liquid diets for $16(n=4), 23(n=4)$, and 30 days $(n=4)$.

Biochemical and histopathological analysis. For measurement of hepatic lipids content, the liver was homogenized at $4^{\circ} \mathrm{C}$ with a Polytron (Kinematica Co., Luzern, Switzerland) homogenizer in buffer composed of $0.25 \mathrm{M}$ sucrose, $50 \mathrm{mM}$ Tris- $\mathrm{HCl}$ ( $\mathrm{pH}$ 7.4), $1 \mathrm{mM}$ EDTA, and centrifuged for $15 \mathrm{~min}$ at $12,000 \mathrm{~g}$. Lipids in the liver homogenate were extracted using chloroform/methanol (1:2 vol/vol), evaporated, and dissolved in 2propanol. The amounts of triglyceride, total cholesterol, and phospholipids were assayed enzymatically, using kits obtained from Wako Pure Chemicals Co. (Tokyo, Japan). Protein was assayed using Protein Assay kit (Bio-Rad Laboratories Inc., Hercules, California, USA). Triglyceride, total cholesterol, phospholipids, total proteins, and albumin in sera were measured, using a standard clinical automatic analyzer (Hitachi 7170; Hitachi Ltd., Tokyo, Japan). Hepatic HGF contents were measured using ELISA as described elsewhere (20) and kits obtained from Institute of Immunology Co. (Tokyo, Japan).

For histological analysis, liver tissues were fixed in 10\% neutralized formaldehyde, embedded in paraffin, and stained with hematoxylin and eosin. Alternatively, hepatic lipids were stained by an oil red $\mathrm{O}$ method (Nacalai Tesque Inc., Kyoto, Japan).

Serum lipoproteins. Very-low-density lipoproteins (VLDL), lowdensity lipoproteins (LDL), and high-density lipoproteins (HDL) in sera were separated using a modification of the method of Hatch and Lees (24). Briefly, VLDL in serum were separated by ultracentrifugation at $114,000 \mathrm{~g}$ for $16 \mathrm{~h}$. After removing the VLDL fraction, density of the remaining serum was adjusted to 1.063 and then ultracentrifuged at $114,000 \mathrm{~g}$ for $20 \mathrm{~h}$. The LDL fraction was removed and density of the remaining serum was adjusted to 1.21 . The remaining serum was ultracentrifuged at $114,000 \mathrm{~g}$ for $40 \mathrm{~h}$, and the HDL fraction was removed.

Preparation of antibodies against apolipoprotein $B(a p o B)$. VLDL and LDL were dialyzed for $48 \mathrm{~h}$ in $0.15 \mathrm{M} \mathrm{NaCl}$ containing 1 mM EDTA. Delipidation of VLDL and LDL was carried out as described (25). Apolipoproteins in VLDL and LDL were dissolved in $1 \%$ SDS, separated by SDS-PAGE, and extracted from the polyacrylamide gel. Female white rabbits were immunized by giving a subcutaneous injection of purified apoB (150-300 $\mu \mathrm{g} / \mathrm{rabbit}$ ) in Freund's complete adjuvant and boosted by injection of the same immunogen with Freund's incomplete adjuvant. IgG was purified from antiserum, using a protein A-Sepharose column.

Measurement of lipids in cultured hepatocytes. Parenchymal hepatocytes were isolated by the in situ collagenase perfusion method, essentially as described elsewhere (26). The isolated hepatocytes were placed on a culture plate coated with type I collagen at a density of $5 \times 10^{5}$ cells $/ \mathrm{ml}$ and cultured for $4 \mathrm{~h}$ in Williams' medium E supplemented with $5 \%$ calf serum, $1 \mathrm{nM}$ insulin, and $1 \mathrm{nM}$ dexamethasone. The culture medium was changed to serum-free Williams' medium E containing $1 \mathrm{nM}$ insulin, $1 \mathrm{nM}$ dexamethasone, and 5 kallikrein inhibitor units $/ \mathrm{ml}$ aprotinin. After culturing the cells in the absence or presence of HGF for $48 \mathrm{~h}$, the cells were washed with ice-cold PBS and then solubilized in lysis buffer composed of $0.5 \%$ SDS, $50 \mathrm{mM}$ Tris- $\mathrm{HCl}$ ( $\mathrm{pH}$ 7.4). Triglyceride in the cell lysate, and in the conditioned medium, was extracted using chloroform/methanol (1:2 vol/vol) and measured in the same manner as described above. VLDL was isolated by ultracentrifugation in the same manner as described above. Viability of cultured hepatocytes was determined by trypan blue exclusion under a phase-contrast microscope, as described elsewhere (19). There was no significant difference in viability of the cells between cultures treated with or without HGF, during $48 \mathrm{~h}$. For histological detection of lipids, the cells were fixed with $4 \%$ paraformaldehyde for $4 \mathrm{~h}$ and subjected to lipid staining, using an oil red $\mathrm{O}$ method.

Pulse-chase analysis of $a p o B$. After culturing hepatocytes in the absence or presence of hrHGF $(20 \mathrm{ng} / \mathrm{ml})$ for $36 \mathrm{~h}$, the cells were washed once with methionine- and cysteine-free DMEM (GIBCO BRL, Gaithersburg, Maryland, USA), and pulse-labeled for $1 \mathrm{~h}$ with $\left[{ }^{35} \mathrm{~S}\right]$ methionine and $\left[{ }^{35} \mathrm{~S}\right]$ cysteine (TranS-label; ICN Biomedicals Inc., Costa Mesa, California, USA) in methionine- and cystine-free medium at $50 \mu \mathrm{Ci} / \mathrm{ml}$. The medium was changed to fresh medium containing $1.5 \mathrm{mg} / \mathrm{ml}$ methionine and $4 \mathrm{mg} / \mathrm{ml}$ cysteine; then the cells were cultured for $5 \mathrm{~h}$. The labeled cells were rinsed twice with $0.5 \mathrm{ml}$ ice-cold PBS. The cells were homogenized in $1 \mathrm{ml}$ of the buffer composed of 0.15 $\mathrm{M} \mathrm{NaCl}, 2 \mathrm{mM}$ EDTA, 20 mM Tris- $\mathrm{HCl}$ ( $\mathrm{pH} 7.4$ ), 1\% sodium deoxycholate, $0.1 \% \mathrm{SDS}, 10 \mu \mathrm{g} / \mathrm{ml}$ aprotinin, $10 \mu \mathrm{g} / \mathrm{ml}$ leupeptin, $10 \mu \mathrm{g} / \mathrm{ml}$ pepstatin, and $1 \mathrm{mM}$ PMSF, and centrifuged at $12,000 \mathrm{~g}$ for $30 \mathrm{~min}$. Cell lysate (1 part) was mixed with the buffer (0.45 part) composed of $0.15 \mathrm{M} \mathrm{NaCl}, 5 \mathrm{mM}$ EDTA, 50 $\mathrm{mM}$ Tris- $\mathrm{HCl}$ ( $\mathrm{pH} 7.4$ ), $0.02 \%$ sodium azide, $0.5 \%$ Triton $\mathrm{X}-100$, $0.5 \%$ sodium deoxycholate, $0.05 \% \mathrm{SDS}, 1 \mathrm{mg} / \mathrm{ml} \mathrm{BSA}$, and proteinase inhibitors, as above, and incubated with $50 \mu \mathrm{l}$ Pansorbin (Calbiochem-Novabiochem Corp., La Jolla, California, USA). After a 5 -h chase, the conditioned medium $(500 \mu \mathrm{l})$ was mixed with $450 \mu \mathrm{l}$ buffer, as above, and incubated with $50 \mu \mathrm{l}$ Pansorbin. After centrifugation at $10,000 \mathrm{~g}$ for $1 \mathrm{~min}$, medium and cell lysate were added to anti-apoB IgG, and the preparation was incubated for $2 \mathrm{~h}$ at $4^{\circ} \mathrm{C}$. The immune complexes were precipitated with $5 \mu \mathrm{l}$ Pansorbin. The immune complexes were separated by SDS-PAGE, visualized by autoradiography, and scanned using a densitometer. The amount of TCA-precipitable ${ }^{35} \mathrm{~S}$-labeled proteins was measured to determine total protein synthesis.

Dot-blot analysis of $a p o B$. Total RNA was prepared from rat liver, as described elsewhere (27), and denatured for $15 \mathrm{~min}$ at $68^{\circ} \mathrm{C}$ in $50 \%$ formamide, $17 \%$ formaldehyde, and $1 \times$ saline sodium citrate (SSC; $0.015 \mathrm{M}$ sodium citrate, $0.15 \mathrm{M} \mathrm{NaCl}[\mathrm{pH}$ 7.0]). Then denatured RNA was blotted on nylon membranes (Amersham Life Sciences Inc., Little Chalfont, United King- 

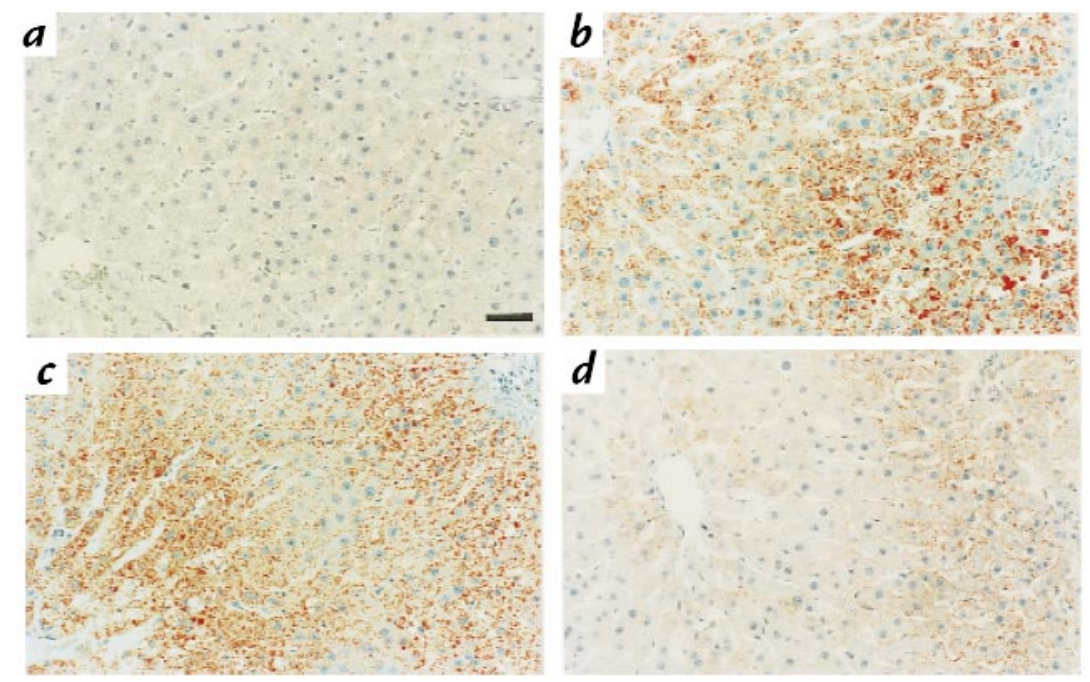

\section{Figure 2}

An accumulation of lipid droplets in the liver of normal and ethanol diet-treated rats, with or without hrHGF administration. Rats were pair-fed isocaloric liquid diets for 30 days $(\boldsymbol{a})$, or ethanol diets for 30 days $(\boldsymbol{b})$ or 37 days $(\boldsymbol{c}$ and $\boldsymbol{d})$. hrHGF $(800 \mu \mathrm{g} / \mathrm{kg})(\boldsymbol{d})$ or saline alone $(c)$ was injected daily for the last 7 days. Liver sections were stained, using an oil red O method. Bars, $50 \mu \mathrm{m}$.

dom) and fixed by ultraviolet irradiation. The membranes were prehybridized and subsequently hybridized with ${ }^{32} \mathrm{P}$-labeled cDNA fragments of rat apoB and rat glyceraldehyde 3-phosphate dehydrogenase (GAPDH). Rat apoB cDNA (a gift from N.O. Davidson, University of Chicago, Chicago, Illinois, USA) was a 660-bp fragment (spanning nucleotides 6,280-6,940) that hybridizes to both apoB100 and apoB48 mRNA (28). Filters were washed under high stringent conditions and subjected to autoradiography. Relative apoB mRNA level was determined by densitometric scanning and normalized to the GAPDH.

Statistical analysis. The paired $t$ test was used to evaluate statistical differences in values between ethanol-containing and isocaloric liquid diet-treated rats. Mann-Whitney $U$ test was used in other experiments.

\section{Results}

Decrease in hepatic lipids after HGF administration. To experimentally develop an ethanol-induced fatty liver, rats were fed ethanol-containing diets for 30 days. In rats given isocaloric pair-fed liquid diets, hepatic triglyceride, total cholesterol, and phospholipids were 44.8, 14.0, and 80.0 $\mu \mathrm{g} / \mathrm{mg}$ protein, respectively. In contrast, in rats fed ethanol-containing diets for 30 days, hepatic triglyceride, total cholesterol, and phospholipids increased to 197.1, 26.9 , and $114.2 \mu \mathrm{g} / \mathrm{mg}$ protein, respectively (Fig. 1). Histological analysis showed that lipid droplets had accumulated in the hepatocytes, preferentially in perivenular and periportal areas, while lipid droplets were rare, even with specific staining of the control liver (Fig. 2, $a$ and $b$ ). Mean body weights of rats given ethanol diets and isocaloric liquid diets were 256 and 264 g, respectively, and liver weights of rats given ethanol diets and isocaloric liquid diets were 8.93 and $8.87 \mathrm{~g}$, respectively. No significant differences were found in body and liver weights between these groups. Therefore, ingestion of ethanolcontaining diets for 30 days led to the onset of fatty liver.

We next asked if HGF would prevent progression or reduce severity of the alcoholic fatty liver. Rats were then fed ethanol-containing diets for 37 days, and these rats were injected daily with saline alone or hrHGF during the last 7 days. In ethanol diet-treated rats given saline alone, hepatic triglyceride content increased to $284.9 \mu \mathrm{g} / \mathrm{mg}$ protein (Fig. 1). In addition, the hepatic total cholesterol and phospholipid levels increased to 48.6 and $131.9 \mu \mathrm{g} / \mathrm{mg}$ protein, respectively. Consistent with this observation, lipid droplets hyperaccumulated in hepatocytes located in perivenular and periportal areas. The lipid accumulation was greater than that seen in rats fed the ethanol-containing diets for 30 days (Fig. 2c). In contrast, in rats injected with hrHGF at $200 \mu \mathrm{g} / \mathrm{kg}$ of body weight for seven days, lipid droplets decreased mainly in perivenular areas (data not shown), and in rats injected with $800 \mu \mathrm{g} / \mathrm{kg}$ hrHGF, accumulation of lipid droplets remarkably decreased in both perivenular and periportal areas (Fig. $2 d)$. Consistent with these results, hepatic triglyceride, total cholesterol, and phospholipids in rats injected with $200 \mu \mathrm{g} / \mathrm{kg}$ hrHGF, respectively, decreased to $70 \%, 72 \%$, and $57.9 \%$ of those in ethanol diet-treated control rats given saline alone. In rats injected with $800 \mu \mathrm{g} / \mathrm{kg}$ hrHGF, triglyceride, total cholesterol, and phospholipids decreased to $27 \%, 48 \%$, and $37 \%$ of those in control rats given saline alone, respectively (Fig. 1). Mean body weights of ethanol diet-treated rats injected with saline, $200 \mu \mathrm{g} / \mathrm{kg}$ hrHGF, and $800 \mu \mathrm{g} / \mathrm{kg}$ hrHGF were 280, 290, and $304 \mathrm{~g}$, respectively, and liver weights were 9.14, 9.56, and $10.27 \mathrm{~g}$, respectively. Significant differences were found in body and liver weights between saline and $800 \mu \mathrm{g} / \mathrm{kg} \mathrm{hrHGF}$ groups $(P<0.05)$. It should be emphasized that hepatic lipids contents in rats given $800 \mu \mathrm{g} / \mathrm{kg}$ hrHGF were lower than in rats treated with ethanol diets for 30 days, even though the rats consumed ethanol diets during the HGF administration. Therefore, HGF administration improved the fatty liver rather than merely suppressing the progression of fatty liver during ethanol diet treatment.

Increases in serum levels of VLDL, $L D L$, and $H D L$. To determine if hrHGF would affect serum lipid concentrations, we next analyzed serum lipid concentrations. Serum concen- 


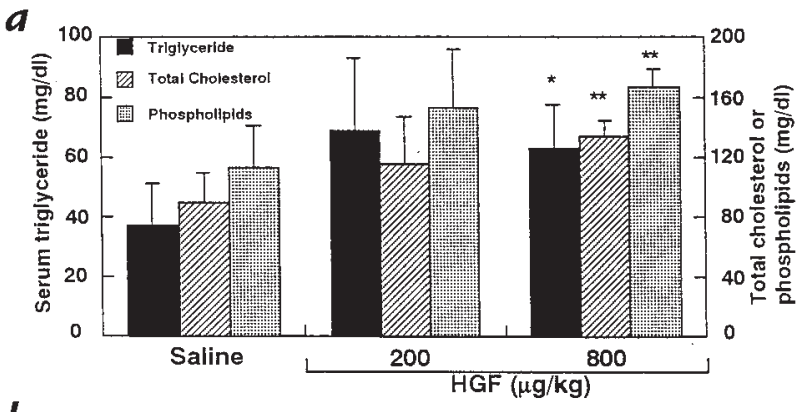

$b$

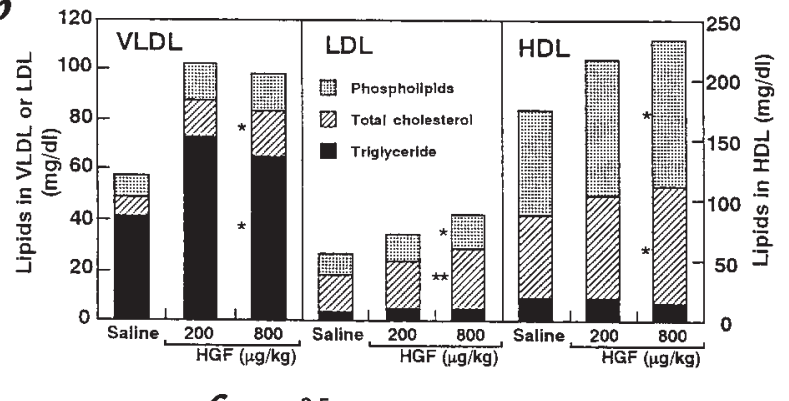

C

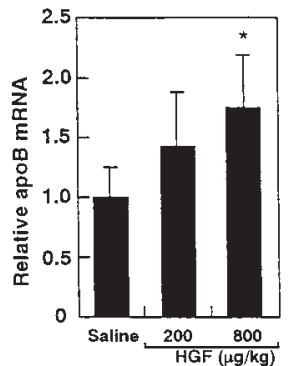

Figure 3

Effects of HGF on serum lipid concentrations and hepatic apoB mRNA levels of rats. Changes in serum lipid concentrations $(a)(n=6)$, lipid compositions in lipoproteins $(\boldsymbol{b})(n=6)$, and hepatic apoB mRNA levels $(\boldsymbol{c})(n=4)$ are shown. Rats were fed ethanol diets for 37 days, and hrHGF or saline alone was daily injected for the last 7 days during 37 days. (c) Total RNA was dot-blotted to nylon membranes and hybridizes with cDNA for apoB and GAPDH, as described in Methods. In $a$ and $c$, each value represents the mean $\pm \mathrm{SD} \ln b$, mean values are represented. ${ }^{*} P<0.05$, ${ }^{* *} P<0.01$ vs. saline control. GAPDH, glyceraldehyde-3-phosphate dehydrogenase.

trations of triglyceride, total cholesterol, and phospholipids in rats injected with $200 \mu \mathrm{g} / \mathrm{kg}$ hrHGF, respectively, increased to 1.8-fold, 1.4-fold, and 1.3-fold higher levels than seen in rats given saline (Fig. $3 a$ ). Likewise, in rats injected with $800 \mu \mathrm{g} / \mathrm{kg}$ hrHGF, serum triglyceride, total cholesterol, and phospholipids, respectively, increased to 1.7-fold, 1.5-fold, and 1.5-fold higher levels than levels in rats given saline (Fig. $3 a$ ). Thus, HGF administration increased serum lipid concentrations in rats with fatty liver; these results suggested that HGF enhanced secretion of lipids from the liver. However, a high dose of HGF (800 $\mu \mathrm{g} / \mathrm{kg}$ ) is more effective in decreasing hepatic triglyceride than a lower dose of HGF $(200 \mu \mathrm{g} / \mathrm{kg})$ (Fig. 1), but the increase in serum triglyceride was saturable at the lower dose (Fig. 3a). Because the serum triglyceride level is mainly regulated by a counterbalance between lipid mobilization from the liver and lipid use in extrahepatic tissues, one possible explanation is that HGF might affect lipid utilization in extrahepatic organs, particularly at higher doses. Although there has been no hard evidence for this, HGF may possibly stimulate subsequent use of lipids in extrahepatic tissues, because HGF stimulates the LDL receptor expression in cultured cells (29) and cell proliferation accompanies biosynthesis of biomembranes. Because 70\% of HGF injected into rats accumulates in the liver (30), HGF in a higher dose seems to exert biological activities in extrahepatic tissues, in case of systemic administration.

Because serum lipids predominantly exist as a lipoprotein complex (i.e., VLDL, LDL, and HDL), we analyzed lipids compositions in VLDL, LDL, and HDL fractions (Fig. 3b). Total lipids recovered in VLDL fraction from rats given 200 and $800 \mu \mathrm{g} / \mathrm{kg}$ hrHGF, respectively, increased to 1.7- and 1.6-fold higher levels than seen in control saline-treated rats. Likewise, triglyceride contents in VLDL from rats given 200 and $800 \mu \mathrm{g} / \mathrm{kg}$ hrHGF, respectively, increased 1.8- and 1.6-fold over those seen in saline-treated rats. These findings suggest that HGF might enhance the secretion of VLDL from the liver and might contribute to improvement in an alcoholic fatty liver. In LDL fractions, administration of hrHGF increased total cholesterol and phospholipids contents in LDL fractions: total cholesterol and phospholipids in LDL fractions from rats injected with $800 \mu \mathrm{g} / \mathrm{kg}$ hrHGF were, respectively, 1.7- and 1.6-fold higher than those seen in saline-treated rats. HDL are cholesterol-rich lipoproteins and transfer cholesterol from peripheral tissues to the liver. Administration of hrHGF increased total cholesterol content in HDL fractions, in a dosedependent manner. hrHGF administration also increased phospholipids content in HDL fractions. Therefore, HGF might affect not only VLDL but also total lipid metabolism, including HDL metabolism.

HGF enhanced lipid secretion by cultured hepatocytes. To observe if the recovery from fatty liver and the stimulation of lipids mobilization from the liver by HGF were exerted through direct effects on hepatocytes, we prepared hepatocytes from rats fed ethanol-containing diets and analyzed lipid mobilization in primary cultures of these hepatocytes. Specific staining clearly indicated that lipid droplets were retained within hepatocytes during the 48-hour culture. However, lipid droplets decreased when the cells were cultured in the presence of $20 \mathrm{ng} / \mathrm{ml}$ HGF (Fig. 4b). Consistent with this, triglyceride contents in hepatocytes cultured in the presence of HGF were 43\% of contents in control cultures (Fig. 4a). These results indicate that HGF directly decreased lipid components that had accumulated within hepatocytes as a result of long-term feeding of ethanol-containing diets.

A previous study indicated that the amount of VLDL in the medium of cultured hepatocytes correlates with the rate of secretion (31). We therefore prepared conditioned medium of hepatocytes cultured in the absence or presence of $20 \mathrm{ng} / \mathrm{ml} \mathrm{HGF}$ for 48 hours and analyzed triglyceride contents in VLDL fractions. In contrast to the decrease in triglyceride contents within hepatocytes by HGF treatment, triglyceride content in VLDL fractions secreted from hepatocytes was 1.9-fold higher than that seen in control (Fig. 4c), which means that HGF directly enhanced the secretion of VLDL from hepatocytes.

Enhancement of apoB synthesis in and secretion by bepatocytes. VLDL secretion is regulated by multiple processes (32), and protein synthesis of apoB is one rate-limiting 
process in VLDL assembly (33). Feeding rats ethanolcontaining diets decreased apoB 100 in VLDL (34). We thus examined the effect of HGF on the synthesis and secretion of apoB, using hepatocytes in primary culture. We prepared hepatocytes from rats fed ethanol-containing diets and cultured with or without $20 \mathrm{ng} / \mathrm{ml} \mathrm{HGF}$ for 36 hours. Newly synthesized proteins were pulse-labeled with $\left[{ }^{35} \mathrm{~S}\right]$ methionine and $\left[{ }^{35} \mathrm{~S}\right]$ cysteine for 1 hour, and then analyzed using sodium dodecyl sulfate polyacrylamide gel electrophoresis (SDS-PAGE) and subsequent radioautography. Newly synthesized apoB100 and apoB48 during the one-hour culture were visible in control cultures, and synthesis of both apoB100 and apoB48 were stimulated by HGF. Because HGF stimulated total protein synthesis by 3.2-fold in cultured hepatocytes, calculation of the rate of protein synthesis of apoB100 and apoB48 per total cellular protein indicated that HGF stimulated the rate of apoB100 and apoB48 synthesis by 2- and 1.5-fold, respectively (Fig. 5).

To determine the mobilization of apoB synthesized during one-hour pulse-labeling, culture medium was changed to $\left[{ }^{35} \mathrm{~S}\right]$ methionine- and $\left[{ }^{35} \mathrm{~S}\right]$ cysteine-free medium, and hepatocytes were further cultured for five hours. ${ }^{35}$ S-labeled apoB100 and apoB48 remaining in the cells and that secreted into the medium were analyzed. Although HGF stimulated both apoB100 and apoB48 protein synthesis, pulse-labeled apoB100 was usually undetectable in the cells, while some pulselabeled apoB48 did remain in the cells. In accordance with the marked decrease in apoB100 and apoB48, secretion of pulse-labeled apoB100 and apoB48 was stimulated by HGF: HGF stimulated secretion of pulse-labeled apoB100 and apoB48 by 3.3- and 1.5 -fold over findings in control culture (Fig. 5). These results indicate that HGF stimulated not only the rate of apoB100 and apoB48 synthesis but also the mobilization of apoB, especially apoB100, presumably as the VLDL complex.

Next, to determine if HGF altered the apoB mRNA level, total RNA was isolated from the liver and change of apoB mRNA level was analyzed by dot-blot hybridization. Densitometric analysis indicated that apoB mRNA levels were increased 1.4- and 1.7-fold by 200 and $800 \mu \mathrm{g} / \mathrm{kg} \mathrm{hrHGF}$ injection, respectively (Fig. 3c). Thus, HGF increases apoB synthesis, at least in part, transcriptionally.

Change of hepatic HGF level during alcoholic fatty change of the liver. The above results indicate that exogenous HGF stimulates lipid mobilization from the liver, and recovery from fatty liver occurs. We therefore analyzed changes in endogenous hepatic HGF levels during the onset of fatty change in the liver (Fig. 6). Rats were fed ethanol-containing diets or pair-fed liquid diets for 16 , 23 , and 30 days. In control rats pair-fed liquid diets for 16,23 , and 30 days, hepatic triglyceride levels were 44.9, 60.4 , and $46.2 \mu \mathrm{g} / \mathrm{mg}$ protein, respectively, whereas endogenous HGF levels were $0.36,0.36$, and $0.40 \mathrm{ng} / \mathrm{mg}$ protein, respectively. In contrast, in rats fed ethanol-containing diets for 16, 23, and 30 days, hepatic triglyceride levels were $53.8,160.5$, and $190.2 \mu \mathrm{g} / \mathrm{mg}$ protein, respectively, whereas endogenous HGF levels were 0.36, 0.51, and $0.66 \mathrm{ng} / \mathrm{mg}$ protein, respectively (Fig. 6). These results indicate that ethanol feeding increased slightly hepatic contents of HGF.
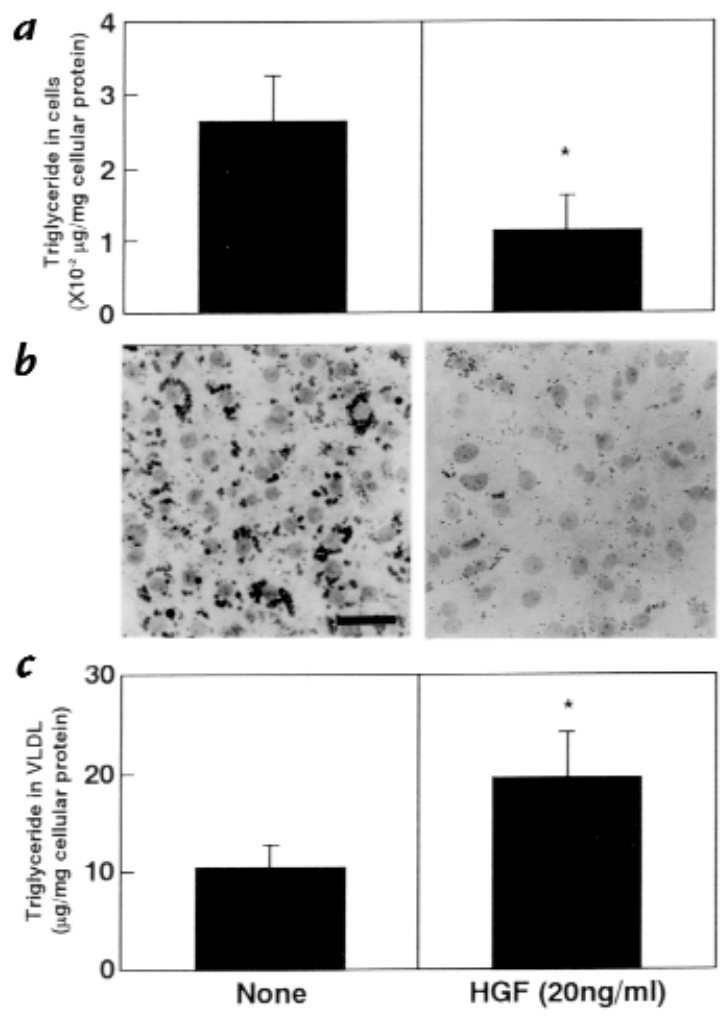

Figure 4

Effects of HGF on lipid mobilization in primary cultured hepatocytes prepared from rat with fatty liver caused by ingestion of ethanol diets. Changes in hepatocellular triglyceride contents $(\boldsymbol{a})$, accumulation of lipid droplets $(\boldsymbol{b})$, and triglyceride levels in secreted VLDL fractions $(\boldsymbol{c})$ are shown. Hepatocytes prepared from rat treated with ethanol diets for 5 weeks were cultured for $48 \mathrm{~h}$ in the absence or presence of $20 \mathrm{ng} / \mathrm{ml} \mathrm{HGF}$. In $b$, lipid droplets in hepatocytes were detected using an oil red $\mathrm{O}$ method. Each value represents the mean \pm SD of triplicate measurements. ${ }^{*} P<0.05$. Bars, $50 \mu \mathrm{m}$. VLDL, very-low-density lipoprotein.

\section{Discussion}

Most lipids derived from adipose tissue and diets are taken up by the liver and synthesized in the liver. The liver plays a major role in regulating lipoprotein levels by virtue of both its degradative and synthetic functions. Based on its predominant roles in systemic lipid metabolism, hepatocellular injuries often lead to alterations in lipid metabolism, and the alcoholic fatty liver is a common disorder accompanying abnormal lipid metabolism. In the present study, we obtained evidence that administration of hrHGF led to a great improvement in the fatty liver. The efficacy of HGF does not seem to be related to the metabolic detoxification of hepatotoxic compounds, because HGF exhibited a direct action in decreasing accumulated lipids in primary cultured hepatocytes. Moreover, HGF injection decreased hepatic lipids contents to levels lower than those seen before HGF administration, even when rats consumed ethanol diets during the period of HGF administration. To our knowledge, this is the first report of amelioration of a fatty liver by a growth factor.

Previous studies indicate that impaired ability of hepatocytes to secrete lipid components as VLDL complex is likely to be one major mechanism linked to an alcoholic 


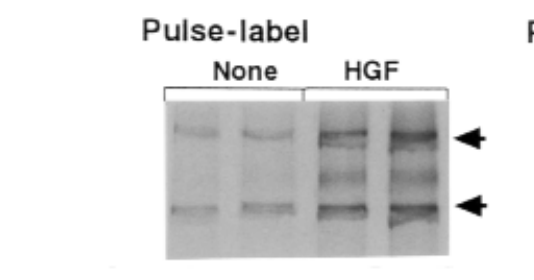

Pulse-chase
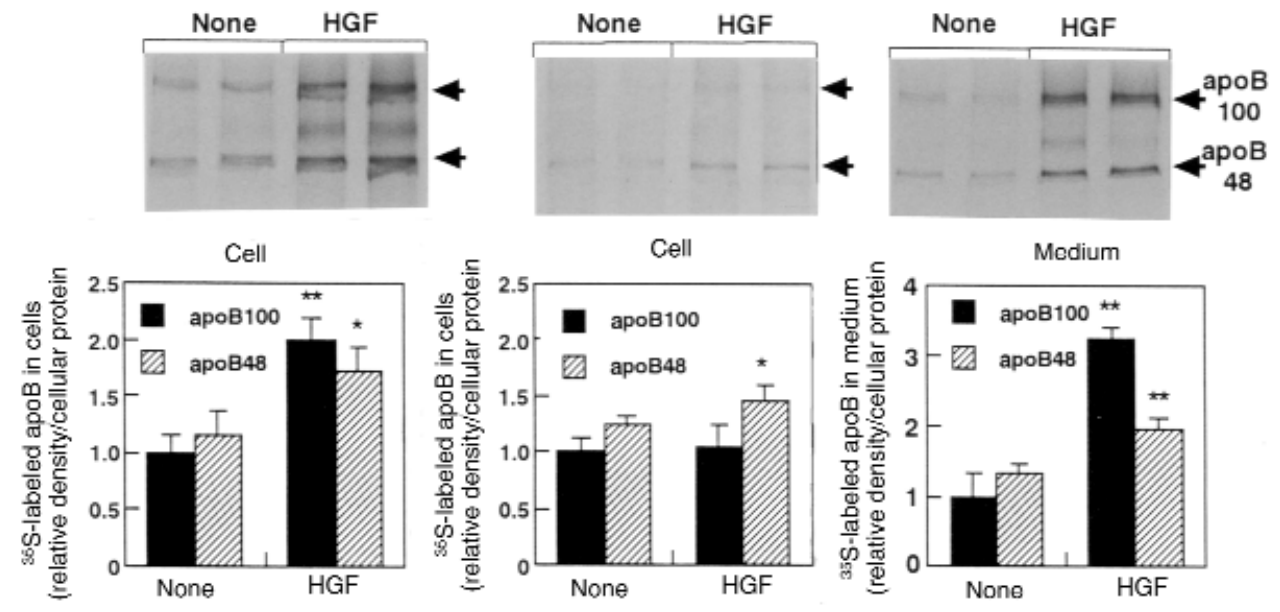

Figure 5

Changes in the rate of apoB synthesis and mobilization of apoB in primary cultured hepatocytes prepared from rat given ethanol diets. Hepatocytes, prepared from rat on ethanol diets for 5 weeks were cultured for $36 \mathrm{~h}$ in the absence or presence of $20 \mathrm{ng} / \mathrm{ml} \mathrm{HGF}$. Cellular proteins were pulselabeled with $\left[{ }^{35} \mathrm{~S}\right]$ methionine and $\left[{ }^{35} \mathrm{~S}\right]$ cysteine for $1 \mathrm{~h}$, apoB was immunoprecipitated from the cell lysate, and immune complexes were subjected to SDS-PAGE. For pulse-chase experiments, after cells were pulse-labeled for $1 \mathrm{~h}$, the cells were cultured for $5 \mathrm{~h}$. ApoB was immunoprecipitated from the cell lysate and from the medium, respectively. Immune complexes were visualized by autoradiography. Lower graphs show changes in synthesis and mobilization of ${ }^{35} \mathrm{~S}$-labeled apoB, as determined by densitometric analysis. Each value represents the mean $\pm \mathrm{SD}$ of triplicate measurements. ${ }^{*} P$ $<0.05,{ }^{* \star} P<0.01$ vs. control.

fatty liver (35-38). Subsequent studies show that hepatic synthesis of apoB is one critical event involved in regulating VLDL secretion (33), and long-term ethanol ingestion suppresses hepatic apoB100 synthesis in rats (34). When we analyzed the rate of apoB synthesis and subsequent mobilization in primary cultured hepatocytes, we found that HGF stimulated both synthesis and the subsequent mobilization of apoB in primary cultured hepatocytes isolated from fatty livers of rats. Consistently,

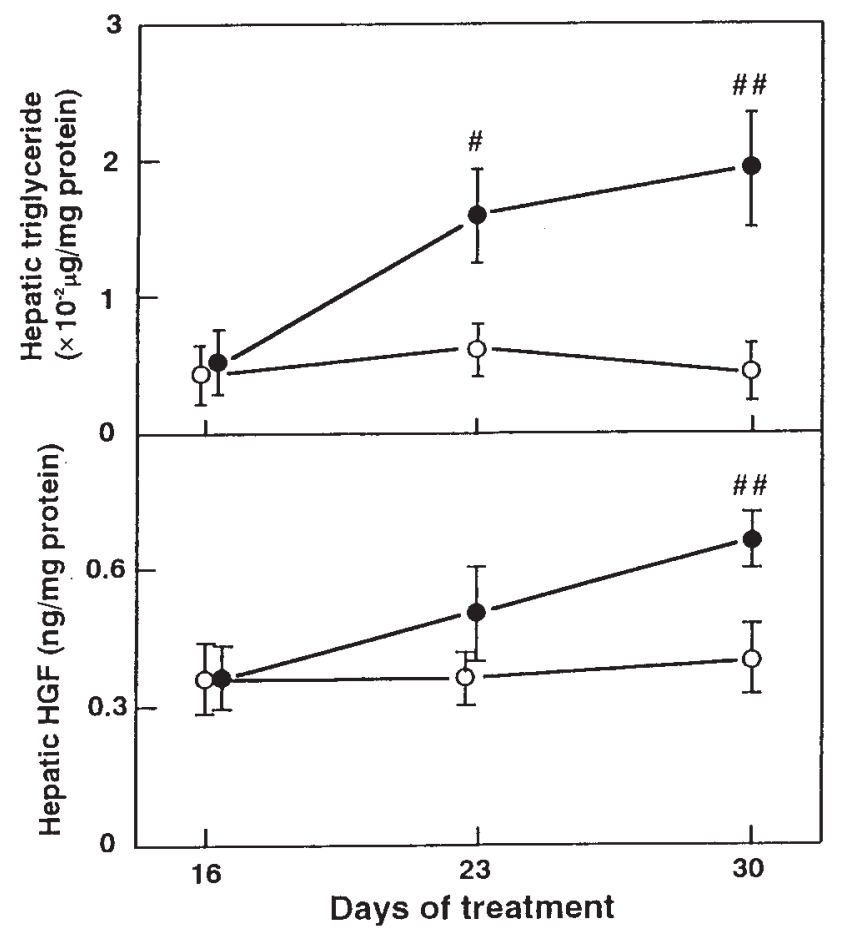

HGF decreased triglyceride contents in hepatocytes with fatty change and stimulated lipid secretion from the cells. Because HGF administration increased serum VLDL levels in rats with fatty livers, in a manner reciprocal to the decrease in hepatic lipid contents, stimulatory effects of HGF on apoB synthesis and subsequent lipid mobilization as VLDL from hepatocytes are, at least, one important mechanism involved in the HGF-induced recovery from an alcoholic fatty liver. Other mechanisms leading to an alcoholic fatty liver include increased fatty acid synthesis and decreased fatty acid degradation (1). Therefore, it may be that HGF has effects on fatty acid metabolism in hepatocytes with alcohol-related fatty change. Previous studies showed that HGF stimulates lipogenesis, including fatty acid synthesis in normal hepatocytes in primary culture (39). Even though HGF stimulates fatty acid synthesis in livers with fatty changes, HGF would stimulate synthesis and subsequent mobilization of lipoproteins and thus lead to recovery from fatty liver.

Physiological and biochemical functions of HGF in regulating hepatic lipid metabolism, in pathological as well as normal livers, remained virtually unknown until recently. Studies showed that HGF stimulates lipid synthesis and secretion in normal hepatocytes and that HGF administration to normal mice increased lipids levels in serum (39-41). These studies suggested a potential role for HGF in regulating lipid metabolism

\section{Figure 6}

Changes in hepatic triglyceride and HGF levels in rats fed ethanol or isocaloric diets. Rats were fed ethanol-containing diets for $16(n=4), 23$ $(n=4)$, and 30 days $(n=4)$ (closed circles), or pair-fed control diets for 16 $(n=4), 23(n=4)$, and 30 days $(n=4)$ (open circles). Each value represents

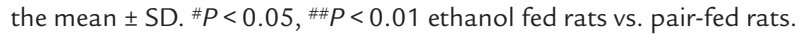


in normal livers; however, expression and level of HGF is low in normal livers $(27,42)$. Therefore, the contribution of HGF in regulating lipid metabolism under normal physiological conditions remains to be addressed. On the other hand, hepatic expression and blood levels of HGF increase in response to acute hepatocellular injuries $(9-12,27,42)$. The endogenously upregulated HGF may support compensatory hepatocyte proliferation and prevent hepatocyte apoptosis. Hepatic expression of HGF in patients with acute alcoholic hepatitis correlates with hepatocyte proliferation and with the survival of patients (43). It is probable that HGF exerts biological activities in regulating lipid metabolism, as well as in stimulating hepatocyte proliferation in acute alcoholic hepatitis. In alcoholic fatty liver, however, there are no obvious hepatocellular injuries nor is there infiltration of inflammatory cells in the liver. Consistent with these general pathological features in alcoholic fatty liver, we found no evidence for inflammatory cells in the liver, and serum levels of glutamic pyruvic transaminase were only slightly increased in the present rat model of alcoholic fatty liver (data not shown). We found that hepatic HGF levels in rats with fatty liver slightly increased, but this increase is much less than in cases of acute hepatic injuries that accompany severe hepatocellular injuries. Endogenous HGF may be insufficient to attenuate disorders in hepatic lipid metabolism because of the alcohol intake.

Physiological roles of HGF have been extensively demonstrated, particularly in liver regeneration. HGF exhibits potent hepatotrophic activity for regeneration of an injured liver $(11,12,14-17)$. HGF has recently been shown to have potent anti-apoptotic activity for hepatocytes (19) and stimulates hepatic protein synthesis, including albumin and fibrinogen $(22,44)$. Thus, HGF exhibits multiple functions in the liver and use of HGF as a therapeutic has been discussed (12). In terms of lipid metabolism, HGF stimulates lipogenesis (39). In rats with alcoholic fatty liver, HGF administration leads to recovery from fatty liver at least, by enhancing apoB protein synthesis and lipid mobilization from the liver. Pak et al. (29) reported that HGF stimulates expression of the LDL receptor in HepG2 cells. Together with these results, HGF is likely to influence hepatic and systemic lipid metabolism through the c-Met/HGF receptor. In addition, it seems noteworthy that HGF administration increased serum HDL levels. Although this increase is not a predominant mechanism for amelioration of fatty liver, our observations do raise the possibility that HGF may have preventive effects regarding arteriosclerotic vascular change, because a drug which targets the liver and enhances serum HDL levels seems to be useful in preventing arteriosclerosis. There are data that HGF is an endothelium-specific growth factor in vascular tissues (45).

Alcoholic fatty liver is a common, chronic hepatic disease, particularly in developed countries. Patients with fatty liver are susceptible to liver fibrosis/cirrhosis $(2,3)$, and increased extracellular matrix synthesis occurs in a fatty liver (4). Other studies noted the efficacy of HGF administration for liver fibrosis/cirrhosis (20-22). Because HGF proved effective in the recovery from a fatty liver in rats, on a one-week treatment with HGF, the potential clinical therapeutic value for subjects with alcohol-related liver disorders warrants further attention.

\section{Acknowledgments}

We are grateful to $\mathrm{M}$. Ohara for critical readings of the manuscript and for useful comments, and to N.O. Davidson for the kind gift of rat apoB cDNA. This work was supported by grants from the Ministry of Education, Science, Sports and Culture of Japan.

1. Nanji, A.A., and Zakim, D. 1996. Alcoholic liver disease. In Hepatology: a textbook of liver disease. 3rd ed. D. Zakim and T.D. Boyer, editors. W.B. Saunders. Philadelphia, PA. 911-913.

2. Sorensen, T.I.A., et al. 1984. Prospective evaluation of alcohol abuse and alcoholic liver injury in men as predictors of development of cirrhosis. Lancet. 2:241-244.

3. Teli, M.R., Day, C.P., Burt, A.D., Bennett, M.K., and James, O.F.W. 1995. Determinants of progression to cirrhosis or fibrosis in pure alcoholic fatty liver. Lancet. 346:987-990. (Abstr.)

4. Feinman, L., and Lieber, C.S. 1972. Hepatic collagen metabolism: effect of alcohol consumption in rats and baboons. Science. 176:795.

5. Mezey, E., Potter, J.J., and Maddrey, W.C. 1974. Hepatic collagen turnover in alcoholic liver disease. Gastroenterology. 67:815. (Abstr.)

6. Nakamura, T., Nawa, K., and Ichihara, A. 1984. Partial purification and characterization of hepatocyte growth factor from serum of hepatectomized rats. Biochem. Biophys. Res. Commun. 122:1450-1459.

7. Russell, W.E., McGowan, J.A., and Bucher, N.L.R. 1984. Partial characterization of hepatocyte growth factor from rat platelets. J. Cell. Physiol. 119:183-192.

8. Nakamura, T., et al. 1989. Molecular cloning and expression of human hepatocyte growth factor. Nature. 342:440-443.

9. Jiang, W.G., Hallett, M.B., and Puntis, M.C.A. 1993. Hepatocyte growth factor/scatter factor, liver regeneration and cancer metastasis. Br. J. Surg. 80:1368-1373.

10. Zarnegar, R., and Michalopoulos, G.K. 1995. The many faces of hepatocyte growth factor: from hepatopoiesis to hematopoiesis. J. Cell Biol. 129:1177-1180.

11. Matsumoto, K., and Nakamura, T. 1996. Emerging multipotent aspects of hepatocyte growth factor. J. Biochem. 119:591-600.

12. Matsumoto, K., and Nakamura, T. 1997. Hepatocyte growth factor (HGF) as a tissue organizer for organogenesis and regeneration. Biochem. Biophys. Res. Commun. 239:639-644.

13. Schmidt, C., et al. 1995. Scatter factor/hepatocyte growth factor is essential for liver development. Nature. 373:699-702.

14. Ishiki, Y., Ohnishi, H., Muto, Y., Matsumoto, K., and Nakamura, T. 1992. Direct evidence that hepatocyte growth factor is a hepatotrophic factor for liver regeneration and has potent antihepatitis effects in vivo. Hepatology. 16:1227-1235.

15. Fujiwara, K., et al. 1993. Stimulation of liver growth by exogenous human hepatocyte growth factor in normal and partially hepatectomized rats. Hepatology. 18:1443-1449.

16. Ishii, T., et al. 1995. Hepatocyte growth factor stimulates liver regeneration and elevates blood protein levels in normal and partially hepatectomized rats. J. Biochem. 117:1105-1112.

17. Roos, F., Terrell, T.G., Godowski, P.J., Chamow, S.W., and Schwall, R.H. 1992. Reduction of alpha-naphtylisothiocyanate-induced hepatotoxicity by recombinant human hepatocyte growth factor. Endocrinology. 131:2540-2544.

18. Shiota, G., Wang, T., Monte, S., Nakamura, T., and Schmidt, E.V. 1994. Hepatocyte growth factor in transgenic mice: effects on hepatocyte growth, liver regeneration and gene expression. Hepatology. 19:962-972.

19. Kosai, K., Matsumoto, K., Nagata, S., Tsujimoto, Y., and Nakamura, T. 1998. Abrogation of Fas-induced fulminant hepatic failure in mice by hepatocyte growth factor. Biochem. Biophys. Res. Commun. 244:683-690.

20. Matsuda, Y., Matsumoto, K., Ichida, T., and Nakamura, T. 1995. Hepatocyte growth factor suppresses the onset of liver cirrhosis and abrogates lethal hepatic dysfunction in rats. J. Biochem. 118:643-649.

21. Yasuda, H., et al. 1996. Antifibrogenic effect of a deletion variant of hepatocyte growth factor on liver fibrosis in rats. Hepatology. 24:636-642.

22. Matsuda, Y., et al. 1997. Preventive and therapeutic effects in rats of hepatocyte growth factor infusion on liver fibrosis/cirrhosis. Hepatology. 26:81-89.

23. Lieber, C.S., DeCarli, L.M., and Sorrell, M.F. 1989. Experimental methods of ethanol administration. Hepatology. 10:501-510.

24. Hatch, F.T., and Lees, R.S. 1968. Practical methods for plasma lipoprotein analysis. Adv. Lipid Res. 6:1-68.

25. Scanu, A.M., and Edelstein, C. 1971. Solubility in aqueous solutions of ethanol of the small molecular weight peptides of the serum very low 
density and high density lipoproteins. Anal. Biochem. 44:576-588.

26. Nakamura, T., Yoshimoto, K., Nakayama, Y., Tomita, Y., and Ichihara, A. 1983. Reciprocal modulation of growth and differentiated functions of mature rat hepatocytes in primary culture by cell-cell contact and cell membranes. Proc. Natl. Acad. Sci. USA. 80:7229-7233.

27. Kinoshita, T., Tashiro, K., and Nakamura, T. 1989. Marked increase of HGF mRNA in non-parenchymal liver cells of rats treated with hepatotoxins. Biochem. Biophys. Res. Commun. 165:1229-1234.

28. Baum, C.L., Teng, B., and Davidson, N.O. 1990. Apolipoprotein B messenger RNA editing in the rat liver. J. Biol. Chem. 265:19263-19270.

29. Pak, Y.K., et al. 1996. Activation of LDL receptor gene expression in HepG2 cells by hepatocyte growth factor. J. Lipid Res. 37:985-998.

30. Liu, K.X., et al. 1992. Importance of the liver in plasma clearance of hepatocyte growth factor in rats. Am.J. Physiol. 263:G642-G649.

31. Davis, R.A., Engelhorn, S.C., Pangburn, S.H., Weinstein, D.B., and Steinberg, D. 1979. Very low density lipoprotein synthesis and secretion by cultured rat hepatocytes. J. Biol. Chem. 254:2010-2016.

32. Dixon, J.L., and Ginsberg, H.N. 1993. Regulation of hepatic secretion of apolipoprotein B-containing lipoproteins: information obtained from cultured liver cells. J. Lipid Res. 34:167-179.

33. Davis, R.A., Boogaerts, J.R., Borchardt, R.A., Malone-McNeal, M., and Archambault-Schexnayder, J. 1985. Intrahepatic assembly of very low density lipoproteins. J. Biol. Chem. 260:14137-14144.

34. Lau, P.P., Cahill, D.J., Zhu, H., and Chan, L. 1995. Ethanol modulates apolipoprotein B mRNA editing in the rat. J. Lipid Res. 36:2069-2078.

35. Schapiro, R.H., Drummey, G.D., Shimizu, Y., and Isselbacher, K.J. 1964. Studies on the pathogenesis of the ethanol-induced fatty liver. II. Effect of ethanol on palmitate-1-C14 metabolism by the isolated perfused rat liver. J. Clin. Invest. 43:1338-1347.
36. Grunnet, N., Kondrup, J., and Dich, J. 1985. Effect of ethanol on lipid metabolism in cultured hepatocytes. Biochem. J. 228:673-681.

37. Venkatesan, S., Ward, R.J., and Peters, T.J. 1988. Effect of chronic ethanol feeding on the hepatic secretion of very-low-density lipoproteins. Biochim. Biophys. Acta. 960:61-66.

38. Day, C.P., and Yeaman, S.J. 1994. The biochemistry of alcohol-induced fatty liver. Biochim. Biophys. Acta. 1215:33-48.

39. Shao, Q., Arakaki, N., Ohnishi, T., Nakamura, O., and Daikuhara, Y. 1996. Effect of hepatocyte growth factor/scatter factor on lipogenesis in adult rat hepatocytes in primary culture. J. Biochem. 119:940-946.

40. Roos, F., Ryan, A.M., Chamow, S.M., Bennett, G.L., and Schwall, R.H. 1995. Induction of liver growth in normal mice by infusion of hepatocyte growth factor/scatter factor. Am. J. Physiol. 268:G380-G386.

41. Kaibori, M., et al. 1998. Hepatocyte growth factor stimulates synthesis of lipids and secretion of lipoproteins in rat hepatocytes. Hepatology. 27:1354-1361.

42. Yamada, A., et al. 1995. Rapid and sensitive enzyme-linked immunosorbent assay for measurement of HGF in rat and human tissues. Biomed. Res. 16:105-114.

43. Fang, J.W.S., Bird, G.L.A., Nakamura, T., Davis, G.L., and Lau, J.Y.N. 1994. Hepatocyte proliferation as an indicator of outcome in acute alcoholic hepatitis. Lancet. 343:820-823.

44. Takehara, T., Matsumoto, K., and Nakamura, T. 1992. Cell densitydependent regulation of albumin synthesis and DNA synthesis in rat hepatocytes by hepatocyte growth factor. J. Biochem. 112:330-334.

45. Nakamura, Y., et al. 1996. Hepatocyte growth factor (HGF) is a novel member of endothelium-specific growth factors: Additive stimulatory effect of HGF with basic fibroblast growth factor, but not vascular endothelial growth factor. J. Hypertens. 14:1067-1072. 